\title{
On the Optimum Number of Cooperating Nodes in Interfered Cluster-Based Sensor Networks
}

\author{
Stefan Mijovic*, Luca Sanguinetti ${ }^{\dagger}$, Chiara Buratti*, and Mérouane Debbah ${ }^{\ddagger \S}$ \\ *DEI, University of Bologna, Bologna, Italy \\ ${ }^{\dagger}$ Dipartimento di Ingegneria dell'Informazione, University of Pisa, Pisa, Italy

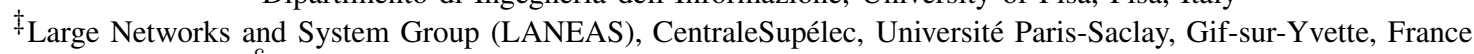

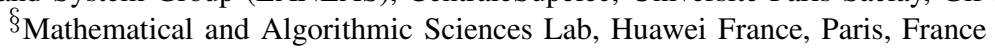

\begin{abstract}
This paper presents a cooperative multiple-input multiple-output (MIMO) scheme for a wireless sensor network consisting of inexpensive nodes, organised in clusters and transmitting data towards sinks. The transmission is affected by hardware imperfections, imperfect synchronisation, data correlation among nodes of the same cluster, channel estimation errors and interference among nodes of different clusters. Within this setting, we are interested in determining the number of nodes per cluster that maximises the energy efficiency of the network. The analysis is conducted in the asymptotic regime in which the number $N$ of sensor nodes per cluster grows large without bound. Numerical results are used to validate the asymptotic analysis in the finite system regime and to investigate different configurations. It turns out that the optimum number of sensor nodes per cluster increases with the inter-cluster interference and with the number of sinks.
\end{abstract}

\section{INTRODUCTION}

Wireless sensor networks (WSNs) have recently gained increasing attention as a practical technology being introduced to different applications. A considerable number of these applications require transmission of the acquired data over long distances using transmission resources available only at sensor nodes. In this situation, direct transmission from a source node to a sink over a fading channel often presents difficulties mainly due to the large amount of energy required to establish a reliable transmission, fostering an inefficient use of batteries.

Multiple-input multiple-output (MIMO) systems are well known for their capability of achieving high spectral efficiency in the presence of fading channels [1]. However, the need to install multiple antennas in sensor nodes can be problematic for economic and practical reasons. To extend the advantages of MIMO systems to single antenna devices, the idea of deploying a cooperative (also known as virtual or distributed) MIMO architecture appears to be very promising. Many works in the literature deal with cooperative schemes for WSNs: one of the first studies was presented in [2], where nodes cooperate to establish virtual antenna arrays; [3] presents a multi-hop cooperative WSN, minimising end-to-end outage probability; in [4] and [5] cooperative beamforming is considered. Despite its promises, the deployment of a cooperative MIMO architecture in WSNs poses several technical challenges mainly because

This research is supported by the FP7 NEWCOM\# (Grant no. 318306). L. Sanguinetti was supported by the ERC StG MORE (Grant no. 305123) and by the research project 5 GIOTTO funded by the University of Pisa. of the large amount of signalling packets required to enable cooperation among different nodes.

Many works in the literature deal with the optimisation of cluster size in non-cooperative WSNs (see, e.g., [6]). Some works address this issue considering cooperative schemes: [5] derives a close-to-optimal number of nodes and a selection method for distributed beamforming; in [7], the optimal cluster size minimizing the outage probability under a Rayleigh fading channel is derived for a cooperative WSN. The aforementioned works, as many others in the literature, do not account for many realistic aspects of WSNs, such as hardware limitation and imperfect synchronisation.

To overcome the issue of overhead in cooperative WSN, this work presents a simple mechanism, which does not require any signalling and is suitable for cheap sensor nodes with limited hardware capabilities. More precisely, we consider a WSN organised in clusters, wherein nodes of each cluster cooperate to transmit data to one or more sinks located in the same cluster [8]. This multi-cluster scenario is analysed by employing the Wyner model [9], which simplifies the analysis and allows for intuitive interpretation of results. Furthermore, in order to facilitate the mathematical computation, we perform the analysis of the asymptotic regime where the number of sensor nodes grows without bound. Results, confirmed by simulations, show that the asymptotic analysis and subsequent optimisation are valid even for relatively small number of sensor nodes.

The main contribution of this work is the analysis and the optimisation of a cooperative WSN affected by inter-cluster interference, imperfect synchronisation and channel estimation, hardware impairments and data correlation within clusters. We provide insights into the most energy efficient cluster configuration, i.e., number of nodes and sinks per cluster, and the system performance under previously mentioned realistic phenomena.

The remainder of this work is organised as follows. ${ }^{1} \mathrm{Sec}-$ tion II describes system and signal models whereas the problem formulation is illustrated in Section III. The asymptotic analysis is presented in Section IV. Numerical results are given in Section V while conclusions are drawn in Section VI.

\footnotetext{
${ }^{1}$ Matrices and vectors are denoted by uppercase boldface and lowercase boldface letters respectively, $\{\cdot\}^{H}$ denotes the Hermitian operator, $\operatorname{tr}\{\cdot\}$ denotes the trace of a matrix, $\operatorname{diag}\{\cdot\}$ denotes a diagonal matrix while $\mathbb{Z}_{+}$ represents the set of all strictly positive integer numbers.
} 

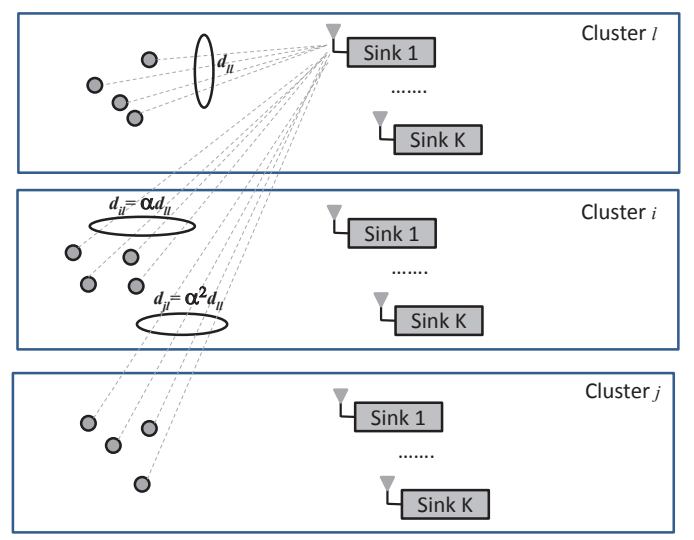

Fig. 1. Reference scenario and Wyner model.

\section{SySTEM AND SIGNAL MODELS}

Consider a WSN composed of $L$ clusters, each consisting of $N$ sensor nodes and $K$ sinks (see Fig. 1). All sensor nodes and sinks are equipped with a single antenna and operate over a bandwidth $B$. A double index notation is used to refer to each node or sink in a given cluster. Under this convention, we refer to node $n i$ as "node $n$ in cluster $i$ " and to sink $k l$ as "sink $k$ in cluster $l$ ".

\section{A. Channel model}

We consider a block flat-fading channel with coherence time $T$ and assume that the transmission takes place according to the time-division duplex (TDD) protocol shown in Fig. 2, with $T_{s}$ being the time required to transmit a symbol and $M$ being the number of symbols transmitted within a frame. As seen, the transmission phase is preceded by a training phase during which pilots, of length $\tau$ symbols, are sent by sinks with $0 \leq \tau \leq M$. Pilots enable sensors to estimate the channels. The TDD protocol is assumed to be matched to the coherence time (i.e., $M T_{s} \leq T$ ). Therefore, the channel can be considered as reciprocal and the sensors can make use of pilot-based estimates for data transmission. Let us denote as $h_{n i k l}$ the channel coefficient between node $n i$ and sink $k l$ and assume that

$$
h_{n i k l}=\sqrt{d_{n i k l}} w_{n i k l}
$$

where $w_{n i k l} \sim \mathcal{C N}(0,1)$ is the small-scale fading channel and $d_{\text {nikl }}$ accounts for the pathloss. For the sake of compactness, we call $\mathbf{h}_{i k l}=\left[h_{1 i k l} \ldots h_{N i k l}\right]^{T}, \mathbf{H}_{i k l}=$ $\operatorname{diag}\left\{h_{1 i k l} \ldots h_{N i k l}\right\}, \mathbf{w}_{i k l}=\left[w_{1 i k l} \ldots w_{N i k l}\right]^{T}$ and $\mathbf{W}_{i k l}=$ $\operatorname{diag}\left\{w_{1 i k l} \ldots w_{N i k l}\right\}$ to denote the channel/fading vector/matrix between all nodes in cluster $i$ and sink $k l$.

To facilitate the analysis, the Wyner model is employed [9]:

- All the nodes within a cluster experience the same pathloss towards the sink antennas, $d_{n l k l}=d_{l l}, \forall n l, k l$;

- All the nodes from the neighbouring cluster experience the same pathloss towards the sink antennas in the observed cluster, $d_{n i k l}=d_{i l}=\alpha d_{l l}, \forall n i, k l$;

- All the nodes from the second neighbouring cluster experience the same pathloss towards the sink antennas in the observed cluster, $d_{n j k l}=d_{j l}=\alpha^{2} d_{l l}, \forall n j, k l$;

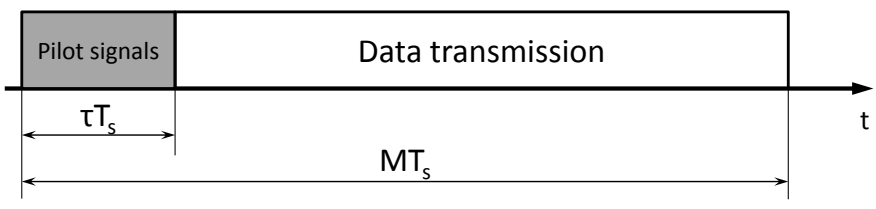

Fig. 2. Time division duplex protocol.

- Interference from all the other clusters is neglected; where $0<\alpha<1$ is the parameter defined by the proximity between the clusters (see Fig. 1).

\section{B. Signal model}

We assume that sensors within a cluster $l$ measure the same parameter such that the transmit data vector $\mathbf{s}_{l}=$ $\left[s_{1 l}, \ldots, s_{N l}\right]^{T}$ has correlation matrix $\mathbf{C}=\mathbb{E}\left[\mathbf{s}_{l} \mathbf{s}_{l}^{H}\right]$, with $[\mathbf{C}]_{n, n}=\mathbb{E}\left[\left|s_{n l}\right|^{2}\right]=1 \forall n$ and $[\mathbf{C}]_{n, m}=\mathbb{E}\left[s_{n l}^{*} s_{m l}\right]=c$ $\forall n \neq m$, where $c$ defines the level of correlation between data of different nodes within the same cluster. On the other hand, data from different clusters are assumed to be uncorrelated.

We denote by $v_{n l}$ the precoding coefficient used by node $n l$ and assume that it is computed as:

$$
v_{n l}=\frac{1}{\sqrt{\lambda_{n l}}} \sum_{k=1}^{K} h_{n l k l}
$$

where $\lambda_{n l}$ is chosen such that the following average constraint $\mathrm{E}\left\{\left|v_{n l}\right|^{2}\right\}=1$ is satisfied. Therefore, from (1) it follows that $\lambda_{n l}=\sum_{k=1}^{K} d_{n l k l}$. In a more compact form, we may write $\mathbf{V}_{l}=\operatorname{diag}\left\{v_{1 l}, \ldots, v_{N l}\right\}$ as

$$
\mathbf{V}_{l}=\boldsymbol{\Lambda}_{l}^{-1 / 2} \sum_{k=1}^{K} \mathbf{H}_{l k l}
$$

with $\boldsymbol{\Lambda}_{l}^{-1 / 2}=\operatorname{diag}\left\{\lambda_{1 l}^{-1 / 2}, \ldots, \lambda_{N l}^{-1 / 2}\right\}$. Let $\hat{\mathbf{V}}_{l}$ be an estimate of $\mathbf{V}_{l}$ and assume that hardware impairments (such as nonlinearities in amplifiers, clock drifts, I/Q imbalance in mixers, finite-precision ADCs and so forth) affect transmission. Similar to [10]-[12], we model the hardware impairments as a reduction of the original signal by a factor $\sqrt{\left(1-\epsilon^{2}\right)}$ (where $\epsilon$ is related to error vector magnitude) and replacing such a loss with Gaussian distortion noise with same power. We denote by $\Phi_{l}$ the matrix that describes imperfect synchronisation among nodes of cluster $l$. Then, the signal received at sink $k l$ takes the form:

$$
y_{k l}=\mathbf{h}_{l k l}^{H} \boldsymbol{\Phi}_{l}\left(\sqrt{p\left(1-\epsilon^{2}\right)} \hat{\mathbf{V}}_{l} \mathbf{s}_{l}+\boldsymbol{\eta}_{l}\right)+\sum_{i=1, i \neq l}^{L} y_{i k l}+n_{k l}
$$

where $p$ is the transmit power, $n_{k l} \sim \mathcal{C N}\left(0, \sigma^{2}\right)$ is the thermal noise while $\boldsymbol{\eta}_{l}=\sqrt{p} \epsilon \hat{\mathbf{V}}_{l} \boldsymbol{\xi}_{l}$ accounts for non-ideal hardware. We assume $\boldsymbol{\xi}_{l} \sim \mathcal{C N}\left(0, \mathbf{I}_{N}\right)$ such that the distortion noise at sensor $n l$ is distributed as $\eta_{n l} \sim \mathcal{C N}\left(0, p \epsilon^{2}\left|\hat{v}_{n l}\right|^{2}\right)$. The term $y_{i k l}$ accounts for the interference generated by cluster $i$ at sink $k l$ given by

$$
y_{i k l}=\mathbf{h}_{i k l}^{H} \boldsymbol{\Phi}_{i}\left(\sqrt{p\left(1-\epsilon^{2}\right)} \hat{\mathbf{V}}_{i} \mathbf{s}_{i}+\boldsymbol{\eta}_{i}\right) .
$$




\section{Problem Statement and Energy-EfFiciency OPTIMISATION}

One of the most common way to define the EE is as a benefit-cost ratio, where the service quality is compared with the associated energy costs. The objective of this work is to solve the following problem:

$$
\max _{N \in \mathbb{Z}_{+}} \mathrm{EE}_{l}=\frac{\left(1-\frac{\tau}{M}\right) \sum_{k=1}^{K} r_{k l}}{P_{\mathrm{T}_{l}}} .
$$

where $r_{k l}$ denotes the achievable rate (in bit/s) at sink $k l$ and $P_{\mathrm{T}_{l}}$ accounts for the total consumed power (in Joule/s) in cluster $l$. The factor $1-\frac{\tau}{M}$ accounts for pilot overhead. The total consumed power is computed as

$$
P_{\mathrm{T}_{l}}=\left(1-\frac{\tau}{M}\right) P_{\mathrm{TX}_{l}}+\frac{\tau}{M} P_{\mathrm{CE}}+N P_{\mathrm{SEN}}+K P_{\mathrm{SINK}}
$$

where $P_{\mathrm{TX}_{l}}$ accounts for the power consumption of the sensor nodes in transmission state, $P_{\mathrm{CE}}$ of the pilot transmission phase whereas $P_{\text {SEN }}$ and $P_{\text {SINK }}$ are constant quantities accounting for the fixed power consumption required by each sensor and sink, respectively, for running the circuitry.

In the sequel, we show how to model and compute all the terms in $\mathrm{EE}_{l}$ when the transmission is affected by intercluster interference, imperfect synchronisation and channel state information (CSI) as well as hardware impairments.

\section{A. Pilot-based Estimation of Precoding Coefficients}

Observe that $d_{n l k l}$ corresponds to the long-term average channel attenuation, which changes in time some orders of magnitudes slower that the fast fading component $w_{n i k l}$. In practice, this means that $d_{n l k l}$ is constant for a sufficiently large number of reception phases to be accurately estimated at the sensor. For this reason, in all subsequent discussions we assume that the quantities $\left\{d_{n l k l} ; \forall k, l\right\}$ are known at sensor $n l$. Therefore, we are only left with the estimation of $\sum_{k=1}^{K} h_{n l k l}$.

In the training phase, we assume sinks of the same cluster to be perfectly synchronised. This can be justified by the fact that sinks can be complex devices employing sophisticated synchronisation mechanisms. Moreover, relatively low number of sinks per cluster guarantees the feasibility of the procedure and limits the overhead. The pilot signal transmitted by sinks in cluster $l$ can be represented by a deterministic vector $\mathbf{u}_{l} \in$ $\mathbb{C}^{\tau \times 1}$ with elements of power $p_{\tau}$. Therefore, we have that $P_{\mathrm{CE}}=K p_{\tau} / \mu_{\mathrm{SINK}}$, where $0<\mu_{\mathrm{SINK}} \leq 1$ accounts for the transceiver efficiency of sinks. We assume that pilot sequences used in different clusters are mutually orthogonal and that the pilot reuse factor is such that the so-called pilot contamination effect is negligible. The collective received signal $\mathbf{x}_{n l} \in \mathbb{C}^{\tau \times 1}$ at sensor $n l$ is given by

$$
\mathbf{x}_{n l}=\sum_{k=1}^{K} h_{n l k l} \mathbf{u}_{l}+\mathbf{n}_{n l}=\nu_{n l} \mathbf{u}_{l}+\mathbf{n}_{n l}
$$

where $\mathbf{n}_{n l} \sim \mathcal{C N}\left(0, \varsigma^{2} \mathbf{I}_{N}\right)$ represents the additive noise at node $n l$ during the pilot signalling. To keep the complexity of nodes at a tolerable level, we employ the least-squares estimator of $\nu_{n l}$ defined as

$$
\hat{\nu}_{n l}=\frac{1}{\tau p_{\tau}} \mathbf{u}_{l}^{H} \mathbf{x}_{n l} .
$$

The variance of the estimation error is given by $\mathrm{E}\left\{\mid \nu_{n l}-\right.$ $\left.\left.\hat{\nu}_{n l}\right|^{2}\right\}=\frac{\varsigma^{2}}{\tau p_{\tau}}$. Plugging (8) into (9) and using (2) yields

$$
\hat{\mathbf{V}}_{l}=\mathbf{V}_{l}+\mathbf{E}_{l}
$$

where $\mathbf{E}_{l} \sim \mathcal{C N}\left(0, \frac{1}{\lambda_{n l}} \frac{\varsigma^{2}}{\tau p_{\tau}} \mathbf{I}_{N}\right)$ is the diagonal estimation error matrix.

Observe that a single pilot signal (i.e., $\tau=1$ ) from all sinks would be sufficient to estimate the precoding coefficients at all sensors. This is a consequence of the adoption of the precoding scheme in (2), which requires sensor $n l$ to have only knowledge of the composite channel $\sum_{k=1}^{K} h_{n l k l}$. Different precoding schemes based on knowledge of $\left\{h_{n l k l} ; k=1, \ldots, K\right\}$ would require $\tau \geq K$. This might not be possible when $K$ is relatively large.

\section{B. Synchronisation Errors}

In any distributed system (such as the one considered in this work), nodes within a cluster cannot be assumed to be perfectly synchronised in time. The cause for imperfect synchronisation ranges from hardware to communication protocol limitations. The transmissions of nodes will be dispersed in time. Considering as a reference time the target time instant of transmission, $t_{0}$, the actual transmission instants of each node can be modelled as a random variable $t_{n l}$, uniformly distributed in range $\left[-t_{\max } / 2, t_{\max } / 2\right]$, where $t_{\max }$ is the maximum synchronisation error represented as a fraction of symbol time $T_{s}$. Assuming an OFDM-based system, i.e., WiFibased WSN, the dispersion in time domain can be represented by phase shift in frequency domain [13] $\phi_{n l}=e^{j 2 \pi t_{n l}}$. The overall effect of synchronisation error is expressed as a complex diagonal matrix $\boldsymbol{\Phi}_{l}=\operatorname{diag}\left\{\phi_{1 l}, \ldots, \phi_{N l}\right\}$ which multiplies the channel matrix $\mathbf{H}_{l l}$. Synchronisation error only introduces phase shift implying that the elements of matrix $\boldsymbol{\Phi}_{l}$ are complex numbers with unit modulus. Matrix $\boldsymbol{\Phi}_{l}$ is unknown to the transmitters, like the channel estimation error, therefore these effects cannot be compensated.

We are interested in observing separately real and imaginary part, $\mathcal{R}\left\{\phi_{n l}\right\}=\cos \left(2 \pi t_{n l}\right)$ and $\mathcal{I}\left\{\phi_{n l}\right\}=\sin \left(2 \pi t_{n l}\right)$. First and second order moments of the two random variables are given by:

$$
\begin{aligned}
\mathbb{E}\left[\mathcal{R}\left\{\phi_{n l}\right\}\right] & =\operatorname{sinc}\left(\mathrm{t}_{\max }\right) \\
\mathbb{E}\left[\left|\mathcal{R}\left\{\phi_{n l}\right\}\right|^{2}\right] & =\frac{1}{2}\left(1+\operatorname{sinc}\left(2 t_{\max }\right)\right)
\end{aligned}
$$

and

$$
\begin{aligned}
\mathbb{E}\left[\mathcal{I}\left\{\phi_{n l}\right\}\right] & =0 \\
\mathbb{E}\left[\left|\mathcal{I}\left\{\phi_{n l}\right\}\right|^{2}\right] & =\frac{1}{2}\left(1-\operatorname{sinc}\left(2 t_{\max }\right)\right) .
\end{aligned}
$$

where $\operatorname{sinc}(x)=\frac{\sin (\pi x)}{\pi x}$. The effect of imperfect synchronisation is twofold: it reduces the useful signal and introduces an interference-like term due to unmatched phases. 


$$
\begin{gathered}
\gamma_{k l}=\frac{\frac{p\left(1-\epsilon^{2}\right) d_{l l}}{K} \mathcal{A}}{\frac{p\left(1-\epsilon^{2}\right) d_{l l}}{K} \mathcal{B}+\frac{p\left(1-\epsilon^{2}\right) d_{l l}}{K} \mathcal{C}(\alpha)+\frac{p}{K} \frac{\varsigma^{2}}{\tau p_{\tau}} \mathcal{D}(\alpha)+\frac{p \epsilon^{2} d_{l l}}{K} \mathcal{E}(\alpha)+\sigma^{2}} \\
\mathrm{EE}_{l}=\frac{\left(1-\frac{\tau}{M}\right) B \sum_{k=1}^{K} \log _{2}\left(1+\gamma_{k l}\right)}{\left(1-\frac{\tau}{M}\right) \frac{p}{\mu_{\text {SEN }}} \frac{1}{K}\left(\operatorname{tr}\left\{\sum_{k=1}^{K} \mathbf{W}_{l k l}^{H} \sum_{k=1}^{K} \mathbf{W}_{l k l}\right\}+\frac{\varsigma^{2}}{\tau p_{\tau}} \frac{N}{d_{l l}}\right)+\frac{\tau}{M} K \frac{p_{\tau}}{\mu_{\text {SINK }}}+N P_{\mathrm{SEN}}+K P_{\mathrm{SINK}}} \\
\bar{\gamma}_{l}(\alpha)=\frac{\left(1-\epsilon^{2}\right) d_{l l} c \operatorname{sinc}^{2}\left(t_{\max }\right)}{\left(1-\epsilon^{2}\right) d_{l l}\left(1-\operatorname{sinc}\left(2 t_{\max }\right)\right)+\frac{\varsigma^{2}}{\tau p_{\tau}}\left(1+\alpha+\alpha^{2}\right)+\epsilon^{2} d_{l l}(K+1)+K d_{l l}\left(\alpha+\alpha^{2}\right)}
\end{gathered}
$$

\section{Energy Efficiency}

Plugging (3) and (10) into (4) and exploiting properties of Wyner model (described in Section II-A) one gets ${ }^{2}$

$$
\begin{aligned}
y_{k l} & =\sqrt{\frac{p\left(1-\epsilon^{2}\right) d_{l l}}{K}} \mathbf{w}_{l k l}^{H} \mathcal{R}\left\{\boldsymbol{\Phi}_{l}\right\} \mathbf{W}_{l k l} \mathbf{s}_{l} \\
& +\sqrt{\frac{p\left(1-\epsilon^{2}\right) d_{l l}}{K}} \mathbf{w}_{l k l}^{H} \mathcal{I}\left\{\boldsymbol{\Phi}_{l}\right\} \mathbf{W}_{l k l} \mathbf{s}_{l} \\
& +\sqrt{\frac{p\left(1-\epsilon^{2}\right) d_{l l}}{K}} \mathbf{w}_{l k l}^{H} \mathbf{\Phi}_{l} \sum_{m=1, m \neq k}^{K} \mathbf{W}_{l m l} \mathbf{s}_{l} \\
& +\sqrt{p\left(1-\epsilon^{2}\right) d_{l l}} \mathbf{w}_{l k l}^{H} \boldsymbol{\Phi}_{l} \mathbf{E}_{l} \mathbf{s}_{l} \\
& +\epsilon \sqrt{p d_{l l}} \mathbf{w}_{l k l}^{H} \mathbf{\Phi}_{l}\left(\sqrt{\frac{1}{K}} \sum_{m=1}^{K} \mathbf{W}_{l m l}+\mathbf{E}_{l}\right) \boldsymbol{\xi}_{l} \\
& +y_{i k l}+y_{j k l}+n_{k l} .
\end{aligned}
$$

The achievable rate at sink $k l$ is thus given by $r_{k l}=$ $B \log _{2}\left(1+\gamma_{k l}\right)$ where $\gamma_{k l}$ is Signal to Interference and Noise Ratio (SINR) computed as in (15) with

$$
\begin{gathered}
\mathcal{A}=\mathbf{w}_{l k l}^{H} \mathcal{R}\left\{\boldsymbol{\Phi}_{l}\right\} \mathbf{W}_{l k l} \mathbf{C} \mathbf{W}_{l k l}^{H} \mathcal{R}\left\{\boldsymbol{\Phi}_{l}\right\} \mathbf{w}_{l k l} \\
\mathcal{B}=\mathbf{w}_{l k l}^{H} \mathcal{I}\left\{\mathbf{\Phi}_{l}\right\} \mathbf{W}_{l k l} \mathbf{C} \mathbf{W}_{l k l}^{H} \mathcal{I}\left\{\mathbf{\Phi}_{l}\right\} \mathbf{w}_{l k l} \\
\mathcal{C}(\alpha)=\mathbf{w}_{l k l}^{H} \boldsymbol{\Phi}_{l} \sum_{m \neq k} \mathbf{W}_{l m l} \mathbf{C} \sum_{m \neq k} \mathbf{W}_{l m l}^{H} \boldsymbol{\Phi}_{l}^{H} \mathbf{w}_{l k l} \\
+\alpha \mathbf{w}_{i k l}^{H} \boldsymbol{\Phi}_{i} \sum_{k=1}^{K} \mathbf{W}_{i k i} \mathbf{C} \sum_{k=1}^{K} \mathbf{W}_{i k i}^{H} \boldsymbol{\Phi}_{i}^{H} \mathbf{w}_{i k l} \\
+\alpha^{2} \mathbf{w}_{j k l}^{H} \boldsymbol{\Phi}_{j} \sum_{k=1}^{K} \mathbf{W}_{j k j} \mathbf{C} \sum_{k=1}^{K} \mathbf{W}_{j k j}^{H} \boldsymbol{\Phi}_{j}^{H} \mathbf{w}_{j k l} \\
\mathcal{D}(\alpha)=\mathbf{w}_{l k l}^{H} \mathbf{w}_{l k l}+\alpha \mathbf{w}_{i k l}^{H} \mathbf{w}_{i k l}+\alpha^{2} \mathbf{w}_{j k l}^{H} \mathbf{w}_{j k l} \\
\mathcal{E}(\alpha)=\mathbf{w}_{l k l}^{H} \sum_{k=1}^{K} W_{l k l} \sum_{k=1}^{K} W_{l k l}^{H} \mathbf{w}_{l k l} \\
+\alpha \mathbf{w}_{i k l}^{H} \sum_{k=1}^{K} W_{i k i} \sum_{k=1}^{K} W_{i k i}^{H} \mathbf{w}_{i k l} \\
+\alpha^{2} \mathbf{w}_{j k l}^{H} \sum_{k=1}^{K} W_{j k j} \sum_{k=1}^{K} W_{j k j}^{H} \mathbf{w}_{j k l}
\end{gathered}
$$

being coefficients depending on fading and synchronisation error only. Regarding the power consumption, starting from (4) and using assumptions from II-A, we obtain

$$
P_{\mathrm{TX}_{l}}=\frac{p}{\mu_{\mathrm{SEN}}} \frac{1}{K}\left(\operatorname{tr}\left\{\sum_{k=1}^{K} \mathbf{W}_{l k l}^{H} \sum_{k=1}^{K} \mathbf{W}_{l k l}\right\}+\frac{\varsigma^{2}}{\tau p_{\tau}} \frac{N}{d_{l l}}\right) \text {. }
$$

where $0<\mu_{\text {SEN }} \leq 1$ accounts for the transceiver efficiency of sensor nodes.

Putting all the above results together, the EE takes the form in (16). At this point one can find EE-optimising $N$ by performing an exhaustive search over the integer set, i.e., performing Monte Carlo simulations of (16) for each possible value of $N$. However, to gain more intuitive insights in the solution of (6) we perform the asymptotic analysis, described in the next section.

\section{Asymptotic AnAlysis}

The analysis is conducted in the regime in which the number of sensors $N$ is infinitely large.

Lemma 1. If $N$ grows without bound, then $\frac{1}{N} \gamma_{l}-\bar{\gamma}_{l} \rightarrow 0$ almost surely with $\bar{\gamma}_{l}$ given by (17). Moreover, we have that $\frac{1}{N} P_{\mathrm{TX}_{l}}-\bar{P}_{\mathrm{TX}_{l}} \rightarrow 0$ almost surely with

$$
\bar{P}_{\mathrm{TX}_{l}}=\frac{p}{\mu_{\mathrm{SEN}}}\left(1+\frac{\varsigma^{2}}{\tau p_{\tau}} \frac{1}{K d_{l l}}\right) .
$$

Proof. The results easily follow using simple statistical arguments and asymptotic results.

Lemma 1 shows that the SINR and the transmit power increase linearly with $N$. Although valid for $N$ growing without bounds, next we use this result for a system with a large but finite number of sensors. This yields

$$
\overline{\mathrm{EE}}_{l}=\frac{\left(1-\frac{\tau}{M}\right) B K \log _{2}\left(1+\bar{\gamma}_{l}(\alpha) N\right)}{\kappa+N \theta_{l}}
$$

where we have defined (for notational compactness)

$$
\theta_{l}=\left(1-\frac{\tau}{M}\right) \frac{p}{\mu_{\mathrm{SEN}}}\left(1+\frac{\varsigma^{2}}{\tau p_{\tau}} \frac{1}{K d_{l l}}\right)+P_{\mathrm{SEN}}
$$

and

$$
\kappa=K P_{\mathrm{SINK}}+\frac{\tau}{M} K \frac{p_{\tau}}{\mu_{\text {SINK }}} .
$$

\footnotetext{
${ }^{2}$ Terms $y_{i k l}$ and $y_{j k l}$ can be expanded in a similar way.
} 
TABLE I

SIMULATION PARAMETERS

\begin{tabular}{|c|c||c|c|}
\hline Parameter & Value & Parameter & Value \\
\hline Intra-cluster distance: $a$ & $100 \mathrm{~m}$ & Transceiver efficiency sensor nodes: $\mu_{\text {SEN }}$ & 0.08 \\
Pathloss model: $d_{l l}$ & $10^{-3.53} a^{-3.76}$ & Transceiver efficiency sinks: $\mu_{\text {SINK }}$ & 0.4 \\
Sensor nodes transmit power: $p$ & $1 \mathrm{~mW}$ & Hardware impairments: $\epsilon^{2}$ & 0.17 \\
Pilot transmit power: $p_{\tau}$ & $100 \mathrm{~mW}$ & Bandwidth: $B$ & $5 \mathrm{MHz}$ \\
Circuit power sensor nodes: $P_{\text {SEN }}$ & $20 \mathrm{~mW}$ & Symbol time: $T_{s}$ & $32 \mu \mathrm{s}$ \\
Circuit power sinks: $P_{\text {SINK }}$ & $100 \mathrm{~mW}$ & Number of symbols in a frame: $M$ & 256 \\
Total noise power: $B \sigma^{2}$ & $-107 \mathrm{dBm}$ & Pilot sequence length: $\tau$ & 1 \\
\hline
\end{tabular}

We now look at the EE-optimal value of $N$ when $\alpha$ is given.

Lemma 2. For $\alpha$ given, the value of $N$ maximising (26) is given by

$$
N^{\star}=\frac{e^{\left(z^{\star}+1\right)}-1}{\bar{\gamma}_{l}}
$$

where

$$
z^{\star}=W\left(\frac{\bar{\gamma}_{l}(\alpha) \kappa}{\theta_{l} e}-\frac{1}{e}\right)
$$

and $W(x)$ is the Lambert function defined by the equation $t=W(t) e^{W(t)}$ for any $t \in \mathbb{C}$.

Proof. The proof relies on using the same augments of Theorem 2 in [14]. Let $\overline{\mathrm{EE}}=\frac{g \log (1+b N)}{c+d N}$ denote the objective function in (26). Note that $\partial \overline{\mathrm{EE}} / \partial N=0$ if and only if

$$
\frac{1}{\ln (2)} \frac{b(c+d N)}{1+b N}-d \log (1+b N)=0
$$

or, equivalently,

$$
\frac{b c-d}{1+b N}=d(\ln (1+b N)-1) .
$$

Plugging $z=\ln (1+b N)-1$ into (32) yields $\frac{b c}{d e}-\frac{1}{e}=z e^{z}$ whose solution is eventually found to be $z^{\star}=W\left(\frac{b c}{d e}-\frac{1}{e}\right)$ where $W(\cdot)$ is the Lambert function. Since $z^{\star}=\ln (1+b N)-$ 1 , the result in (29) follows.

A close inspection of (29) reveals that $N^{\star}$ increases with $\alpha$. This is because higher interference level brings to lower SINR, with ensuing reduction of the achievable rate. This can only be compensated by increasing $N^{\star}$. Another interesting observation is that $N^{\star}$ must increase with $K$. This is due to the fact that when $K$ increases, the energy consumption of sinks becomes the dominant component of the overall energy consumption, due to the high transmit power of pilots and complex circuitry of sinks $\left(P_{\text {SINK }}\right)$. In this setting, increasing $N$, while being beneficial for the achievable rate, does not affect the total energy consumption too much, implying that EE-optimal $N$ should be higher.

\section{NUMERICAL RESULTS}

Monte Carlo simulations have been used to validate the analysis above. Results are obtained averaging over 1000 realisations of all the random variables, such as fading, noise, etc. The network parameters are given in Table I. Those related to the energy consumption are taken from the datasheet of a popular WSN device, TI CC2530 [15], while PHY layer parameters are inspired by the IEEE 802.15.4 standard [16]. Unless otherwise specified, the level of correlation of data within a cluster is $c=0.8$ and the maximum synchronisation error is $t_{\max }=0.5$ (symbol time).

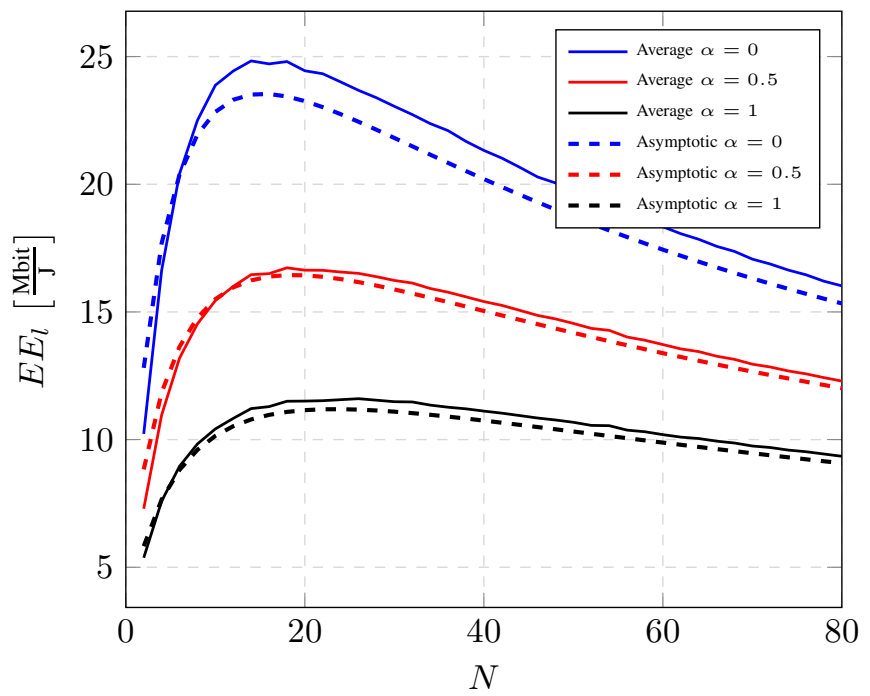

Fig. 3. Energy efficiency vs. $N$ for $K=3$ and $\alpha=0,0.5$ and 1 .

Fig. 3 plots the energy efficiency as a function of $N$ for $K=3$ and different values of $\alpha$. The curves obtained from the asymptotic results of Lemma 1 closely follow the ones obtained through Monte Carlo simulations. This proves that the asymptotic analysis is accurate even for a relatively low number of sensor nodes. As expected, the energy efficiency decreases with $\alpha$ due to the higher level of interference.

Fig. 4 illustrates the EE-optimal value of sensor nodes as a function of $\alpha$ for $K=1,3$ and 5. Firstly, notice that the closed form solution obtained through (29) is very close to the values obtained through simulations. The difference between the two is higher for higher values of $K$. However, for high values of $K$, i.e., $K \geq 5$, a wider range of values of $N$ achieves similar energy efficiency (see Fig. 5) such that $N^{\star}$ computed by (29) achieves energy efficiency very close to the optimal one. Secondly, as predicted at the end of Section IV, $N^{\star}$ increases with $\alpha$ and $K$.

Fig. 5 reports energy efficiency as a function of $N$ for $\alpha=0.5$ and different values of $K$ and $t_{\max }$. We can observe a large performance gap between the case where synchronisation is perfect, $t_{\max }=0$, and the case where the maximum 


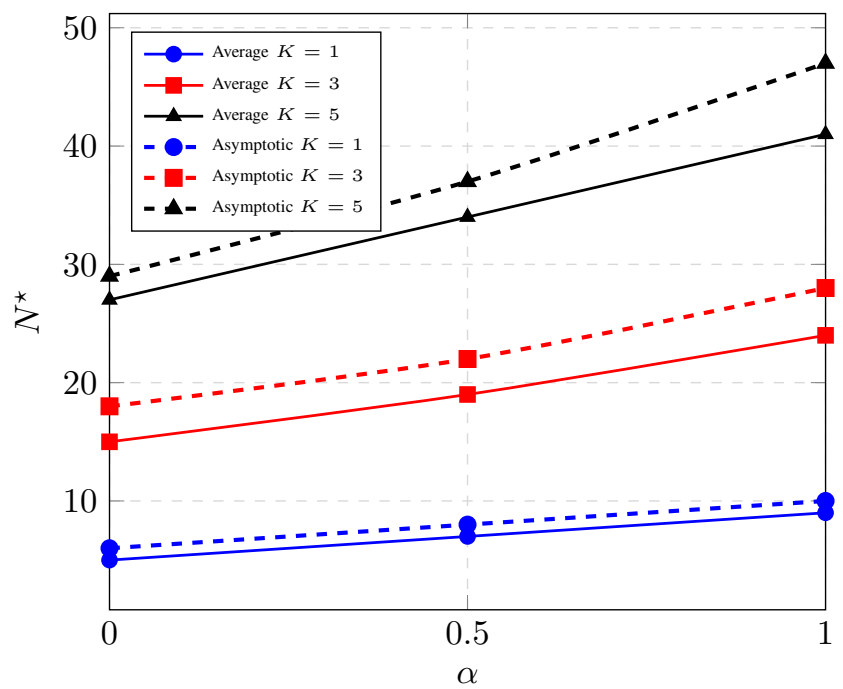

Fig. 4. EE-optimal value of sensor nodes $N^{\star}$ vs. $\alpha$ for $K=1,3$ and 5 .

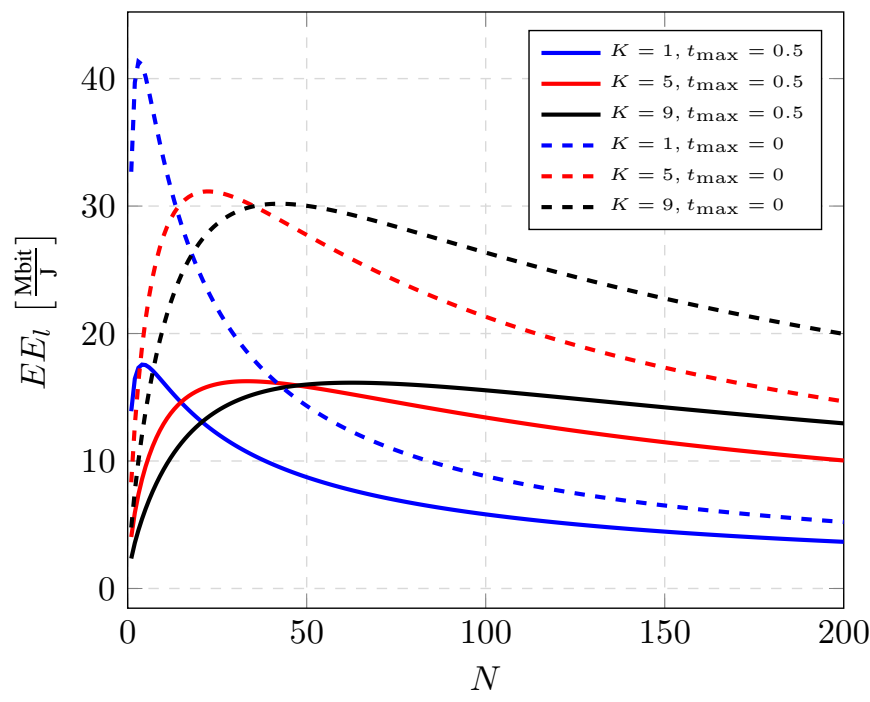

Fig. 5. Energy efficiency vs. $N$, for $\alpha=0.5$ and different $K$ and $t_{\max }$.

synchronisation error is $t_{\max }=0.5$. The global maximum of the energy efficiency is obtained for $K=1$, while the value of $N^{\star}$ depends on the level of desynchronisation among nodes. Fig. 5 also gives an insight about the solution of the reverse problem: if $N$ is given, find the EE-maximising value of $K$. These remarks would not change for other values of $\alpha$ and $c$.

\section{CONCLusions}

In this paper, we focused on the maximisation of the energy efficiency in a cooperative MIMO scheme for WSN in which low-cost sensor nodes are organised in clusters and transmit data to sinks. Wyner model was considered for analytic tractability. The analysis took into account hardware limitations that typically affect low-cost devices, imperfect synchronisation and data correlation among nodes of the same cluster, imperfect CSI as well as inter-cluster interference. The asymptotic analysis was used to compute a closed form expression for the EE-optimal number of nodes per cluster, $N^{\star}$. This allowed to get some insights on how $N^{\star}$ is affected by the network parameters. In particular, it turned out that $N^{\star}$ must increase with the inter-cluster interference level as well as with the number of sinks in each cluster. We also provided an insight on how imperfect synchronisation affects the performance, however this aspect is yet to be analysed in details. Monte Carlo simulations were eventually used to validate the asymptotic analysis, which is proved to be accurate even for a relatively low number of sensor nodes.

\section{REFERENCES}

[1] E. Telatar, "Capacity of Multi-antenna Gaussian Channels," European Transactions on Telecommunications, vol. 10, no. 2, p. 585595, Autumn 1999.

[2] M. Dohler, A. Gkelias, and H. Aghvami, "A resource allocation strategy for distributed MIMO multi-hop communication systems," IEEE Communications Letters, vol. 8, no. 2, pp. 99-101, Feb 2004.

[3] A. Del Coso, U. Spagnolini, and C. Ibars, "Cooperative distributed mimo channels in wireless sensor networks," Selected Areas in Communications, IEEE Journal on, vol. 25, no. 2, pp. 402-414, 2007.

[4] G. Barriac, R. Mudumbai, and U. Madhow, "Distributed beamforming for information transfer in sensor networks," in Third International Symposium on Information Processing in Sensor Networks, April 2004, pp. 81-88.

[5] N. Tessema, X. Lian, and H. Nikookar, "Distributed beamforming with close to optimal number of nodes for green wireless sensor networks," in Online Conference on Green Communications (GreenCom), 2012 IEEE, Sept 2012, pp. 139-144.

[6] H. Abusaimeh and S.-H. Yang, "Energy-aware optimization of the number of clusters and cluster-heads in wsn," in Innovations in Information Technology (IIT), 2012 International Conference on, March 2012, pp. 178-183.

[7] K. Shin, W.-C. Kim, S.-J. Park, and D.-H. Cho, "Cooperative communication with joint optimization of cluster size and resource allocation in wireless sensor networks," in MILITARY COMMUNICATIONS CONFERENCE, 2012 - MILCOM 2012, Oct 2012, pp. 1-5.

[8] J. Agrakhed, G. Biradar, and V. Mytri, "Cluster based energy efficient qos routing in multi-sink wireless multimedia sensor networks," in Industrial Electronics and Applications (ICIEA), 2012 7th IEEE Conference on, July 2012, pp. 731-736.

[9] A. Wyner, "Shannon-theoretic approach to a Gaussian cellular multipleaccess channel," Information Theory, IEEE Transactions on, vol. 40, no. 6, pp. 1713-1727, Nov 1994.

[10] W. Zhang, "A General Framework for Transmission with Transceiver Distortion and Some Applications," IEEE Transactions on Communications, vol. 60, no. 2, pp. 384-399, Feb. 2012.

[11] E. Bjornson, P. Zetterberg, M. Bengtsson, and B. Ottersten, "Capacity Limits and Multiplexing Gains of MIMO Channels with Transceiver Impairments," IEEE Communications Letters, vol. 17, no. 1, pp. 91-94, Jan. 2013

[12] E. Björnson, J. Hoydis, M. Kountouris, and M. Debbah, "Massive MIMO Systems With Non-Ideal Hardware: Energy Efficiency, Estimation, and Capacity Limits," IEEE Transactions on Information Theory, vol. 60 , no. 11, pp. 7112 - 7139, Nov 2014.

[13] H. Balan, R. Rogalin, A. Michaloliakos, K. Psounis, and G. Caire, "AirSync: Enabling Distributed Multiuser MIMO With Full Spatial Multiplexing," IEEE/ACM Transactions on Networking, vol. 21, no. 6, pp. 1681-1695, Dec 2013.

[14] E. Bjornson, L. Sanguinetti, J. Hoydis, and M. Debbah, "Optimal design of energy-efficient multi-user MIMO systems: Is massive MIMO the answer?" IEEE Trans. Wireless Commun., vol. 14, no. 6, pp. $3059-$ 3075, June 2015.

[15] "Texas Instruments CC2530 - Second Generation System-on-Chip Solution for 2.4 GHz IEEE 802.15.4 / RF4CE / ZigBee ," http://www.ti. $\mathrm{com} /$ product/cc2530.

[16] "IEEE Standard for Local and metropolitan area networks-Part 15.4: Low-Rate Wireless Personal Area Networks (LR-WPANs)," http:// standards.ieee.org/getieee802/download/802.15.4-2011.pdf, 2011. 